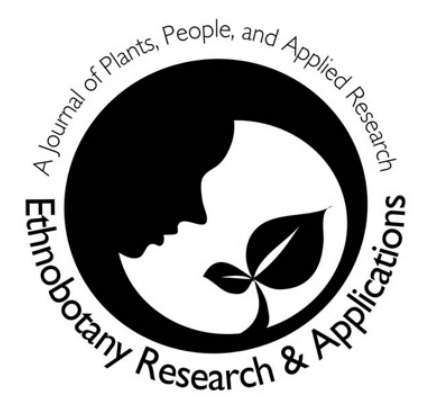

\title{
Ethnobotanical inventory and therapeutic applications of plants traded in the Ho Central Market, Ghana
}

Maxwell Kwame Boakye, Alfred Ofori Agyemang, Bernard Kofi Turkson, Edward Debrah Wiafe, Michael Frimpong Baidoo and Marcel Tunkumgnen Bayor

\section{Correspondence}

Maxwell Kwame Boakye ${ }^{1^{*}}$, Alfred Ofori Agyemang ${ }^{2}$, Bernard Kofi Turkson ${ }^{3}$, Edward Debrah Wiafe ${ }^{4}$, Michael Frimpong Baidoo², and Marcel Tunkumgnen Bayor ${ }^{2}$

${ }^{1}$ Department of Environmental Science, Ho Technical University, Ho, Ghana

${ }^{2}$ Institute of Traditional and Alternative Medicine, University of Health and Allied Sciences, Ho, Ghana

${ }^{3}$ Department of Herbal Medicine, Kwame Nkrumah University of Science and Technology, Kumasi, Ghana

${ }^{4}$ School of Natural and Environmental Sciences, University of Environment and Sustainable Development, Somanya, Ghana

*Corresponding Author: mboakye@htu.edu.gh

Ethnobotany Research and Applications 23:8 (2022)

\section{Research}

\begin{abstract}
Background. For therapeutic purposes, local markets serve as the main trading center of medicinal plants for both traditional medicine practitioners and the general public. However, there is still limited information about the plant species and their derivatives traded outside larger markets in major cities. This study aimed to take inventory of the plant species traded for medicinal purposes in a traditional market outside a major city and determine their applications through vendors' familiarization.
\end{abstract}

Methods: An inventory of plant species traded for medicinal purposes in the Ho Central Market was undertaken, coupled with a semi-structured interviews on their applications based on the vendor's familiarization. Quantitative ethnobotanical indices were used to determine the most culturally important species.

Results. A total of 60 plant species from 37 families was documented to be traded in the Ho Central Market. The Adansonia digitata, Thaumatococcus daniellii and Zanthoxylum zanthoxyloides were recorded to have the highest frequency of citations. The highest use report (UR) and cultural importance $(\mathrm{Cl})$ values were recorded for $Z$. zanthoxyloides. Body pains, blood tonic (anemia), and abdominal pains were the most prominent conditions that traded plants were used to treat.

Conclusion: The study revealed a total of 60 medicinal plants which is commonly sold in the Ho Central Market and other major markets in the country, which was attributed to idiosyncrasy in cultural knowledge about the application of the plants. The market served as a venue for information exchange and learning, resulting in the high uniformity of vendors' familiarization with plant application and that of traditional medicine practitioners.

Keywords. Local market, Medicinal plants, Trade, Cultural importance, Traditional medicine, Body pains 


\section{Background}

Local markets serve as the main trading center for goods and services and are a source of medicinal plants for both traditional medicine practitioners and the general public for therapeutic purposes. These markets serve as a concentration and diffusion center for empirical knowledge about plant resources, which helps maintain local knowledge about medicinal plants (Bye \& Linares 1983; Albuquerque et al. 2007; Monteiro et al. 2010). The local markets provide a snapshot of the plant biodiversity of a region (Albuquerque et al. 2007; van Andel et al. 2007, 2012; Ouedraogo et al. 2020;). Market surveys have become an indispensable aspect of understanding the supply and the demand for medicinal plants and are instrumental in identifying species requiring resource management priorities (Martin 1995; Albuquerque et al. 2007; van Andel et al. 2012; Bussmann et al. 2016, 2018; Ouedraogo et al. 2020; Oliveira et al. 2021).

In Ghana, ethnobotanical market survey studies have provided insights into the use of botanical resources and plant diversity through trade (Obiri \& Addai 2007; Asase \& Oppong-Mensah 2009; van Andel et al. 2012). However, previous market surveys were conducted in traditionally established larger markets in major cities, creating a paucity of information for smaller cities not known for their large markets. The Volta Region is rich in plant diversity because the vegetation is a mixture of both forest and savanna species but not known for its larger markets, leading to under-documentation regarding the sale of plants for traditional medicine purposes. Currently, no known ethnobotanical market survey has been undertaken in any of the traditional markets in the region.

Market surveys efficiently acquire data on local consumption and conservation status of medicinal plant species (Idu et al. 2010; Ouedraogo et al. 2020). Markets provide a shortlist of the much more comprehensive range of species used in a country or a locality (van Andel et al. 2007). The market also provides insight into indigenous uses of plants that contribute to preserving indigenous knowledge, which is disappearing at an alarming rate (Ouedraogo et al. 2020). Studies of traditional markets are necessary for Ghana to gain insights into the plant species traded and their impact on plant populations because of the significant gaps in knowledge on flora trade outside major cities. There is a need to investigate the medicinal plants sold in relatively minor markets to determine the medicinal plant species sold and their relation to local health disorders. Identified medicinal plants may also serve as leads in the research and discovery of medications from natural sources. This study aimed to provide an inventory of plant species traded for medicinal purposes in the Ho Central Market, one of the oldest traditional markets in the Volta Region but without any information on ethnobotanical trade. The study objectives were to (1) document the medicinal plants commercialized in the Ho Central market, (2) determine whether traded plant species are comparable to those of larger markets in major cities, and (3) determine their therapeutic applications based on vendors' familiarization.

\section{Materials and Methods}

\section{Study area}

The study was conducted in the Ho Municipal area in the Volta Region of Ghana (Figure 1). The study area is located between $6^{\circ} 20^{\prime \prime} \mathrm{N}$ and $6^{\circ} 55^{\prime \prime} \mathrm{N}$ latitudes $0^{\circ} 12^{\prime} \mathrm{E}$ and $0^{\circ} 53^{\prime} \mathrm{E}$ longitude and covers an area of approximately 2,361 $\mathrm{km}^{2}$ (Ghana Statistical Service 2014). It shares boundaries with Ho West District to the North and West, Adaklu and Agotime-Ziope Districts to the South and Republic of Togo to the East. Based on the 2010 Population and Housing Census report, the human population of Ho Municipal is estimated at around 177281 (Ghana Statistical Service, 2014). Ho city is regarded as the center of commerce in the Volta Region as it serves as the regional capital. There is a deep-rooted belief in the potency of plant materials and the high patronage of traditional medicine in the municipality (Konlan et al. 2020). There is no known ethnobotanical inventory of local markets in the study area based on a literature search, making it ideal for conducting this study. In addition, this market serves as a converging point for all the satellite markets in the adjoining towns.

\section{Sampling method and data collection}

There are three (3) main markets in Ho city: Ho Central Market, Ahoe Market and Civic Centre Market. A preliminary survey of these three main markets revealed that plants sale only occurs at the Ho Central Market for traditional medicine purposes. The queen of the market was first informed about the nature, scope, and aim of the research and obtain informed consent to research in the market. An informed consent process made all the participants aware of their rights to decide to participate voluntarily or decline after the purpose of the research had been made known to them. Interviews were conducted only after obtaining participants' prior informed consent. This research was conducted following the International Society for Ethnobiology Code of Ethics that provides a framework for 
conducting ethnobiological research and related activities (International Society of Ethnobiology 2006). No further ethics approval was required.

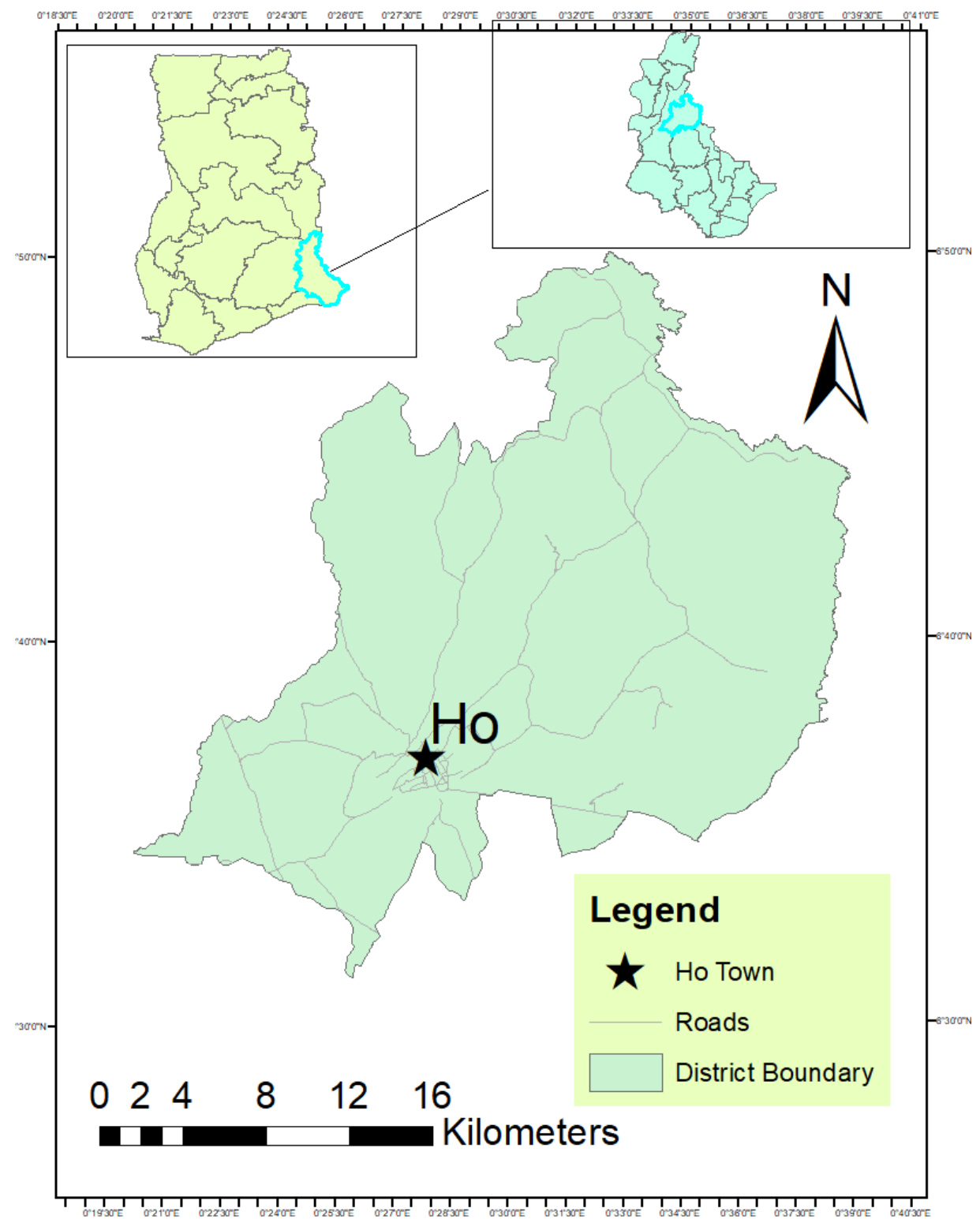

Figure 1. Map of Ghana indicating the Volta Region and Ho Municipal

An inventory was conducted on plants traded for traditional medicinal purposes in the Ho Central Market in November and December 2020. The sampling approach was purposeful, with only vendors selling medicinal products selected because they could provide the information needed for this study. All 22 vendors identified as selling plants and their derivatives for medicinal purposes agreed to partake in this study. Data were gathered using a semi-structured interview guide that was supplemented by an inventory of the plant species displayed. The interview questions were guided by Martin (1995) suggested lines of inquiry for local markets research. The key questions put to the vendors included the plant products offered for sale, their familiarization with the therapeutic applications of plant species and parts sold, condition of the goods (fresh or dried), management, and marketing of the resource (cultivated or wild). Within the data collection period, no changes were observed concerning changes in the inventoried plant species. The interviews were conducted in the Ewe language, the most widely spoken language in the study area for those who could not speak English. Maxwell Kwame Boakye and Alfred Ofori Agyemang, together with the field assistant (Mathias Gidisu), conducted the interviews with all the participants. The field assistant was fluent in the Ewe language and did most of the Ewe translation. Most of the questions were structured to avoid loss of meaning through the translation. 
Plant species identification was carried out by matching the local names mentioned by the vendors with that in the available literature (Irvine, 1961; Abbiw, 1990; Mshana et al. 2000). The collection of plant materials was used to confirm their identification by comparison with voucher specimens at the Institute of Traditional and Alternative Medicine (ITAM), University of Health and Allied Sciences (UHAS). The names and authority of plant species were confirmed using electronic databases (http://www.worldfloraonline.org and http://www.theplantlist.org).

\section{Data analysis}

Ethnobotanical analysis was performed with the ethnobotanyR package in R (Cory, 2020). The ethnobotany package was used to calculate common quantitative ethnobotanical indices. The calculated indices included: Use report (UR), Cultural importance (Cl), Frequency of citation (FC), Number of uses (NU), Relative frequency of citation (RFC), Relative importance index (RI), and fidelity level (FL) per species.

The UR values per species count the number of informants who mention each use category for the species and the sum of all uses in each use category (Prance et al. 1987). The number of uses (NU) per species is the sum of all categories considered useful for a species (Prance et al. 1987). The cultural importance $(\mathrm{Cl})$ index calculates the cultural importance index for each species in the data set (Tardío \& Pardo de Santayana, 2008). The frequency of citation (FC) per species is the sum of informants that cite a use for the species in the dataset (Prance et al. 1987). The relative frequency of citation (RFC) determines the significance of every species based on the number of informants who reported using it. The relative importance (RI) index calculates the relative importance for each species in the data set, considering only the use categories (Tardío \& Pardo de Santayana, 2008). The fidelity level $(\mathrm{FL})$ per species calculates the percentage of informants who use a plant for the same purpose compared to all plants' uses (Friedman et al. 1 986).

A word cloud visual representation of word frequency was used to get instant insight into plant species' most prominent or prevalent applications based on vendors' familiarization using Wordlt Out. The sizes of the words are proportional to the frequency with which the vendors mentioned the words. A visualization of flows i.e., weighted connections between plant parts sold and their condition was presented in a Sankey diagram using Power BI.

\section{Results}

\section{Composition of commercialized plants and their characteristics}

A total of 60 plant species belonging to 37 families were documented to be traded in the Ho Central Market (Table 1). In the total of 37 families of plants, members of the Fabaceae had the highest percentage of plants with $8.3 \%$ ( $n=5$ species), followed by Apocynaceae and Malvaceae with $6.7 \%(n=4$; each), Cucurbitaceae and Zingiberaceae with 5\% ( $n=3$ species; each), Amaryllidaceae, Annonaceae, Apiaceae, Compositae, Euphorbiaceae, Lamiaceae, Rubiaceae, Rutaceae, and Solanaceae had 3\% ( $n=2$ species; each). While the remaining families contributed only one species, each $1.7 \%$ (Table 1 ).

Based on plant habit, herbaceous species were commonly cited with $45 \%$ ( $n=26$ species) followed by trees with $38.33 \%$ ( $n=23$ species), and shrubs with $16.66 \%$ ( $n=10$ species). With regard to management and marketing of the resource (cultivated or wild) of the plants inventoried, wild forms contributed to $45 \%$ ( $n=27$ ), cultivated forms to $16.66 \%(n=10)$ and both (wild/cultivated) contributed to $38.33 \%(n=23)$. Khaya senegalensis and Garcinia kola are classified as vulnerable species under the IUCN Red List categories of species while the remaining were classified either as Least Concern or Data Deficient.

The plant parts such as Fruits, stem bark, roots, leaves, seeds, aerial parts, bulbs, flowers, rhizome, and whole plant were documented to be sold in the Ho Central Market. Fruits were the most common commercialized plant part with $32.31 \%$ followed by stem bark $(16.92 \%)$, roots $(13.85 \%)$, leaves $(9.23 \%)$, seeds and aerial parts $(7.69 \%$; each), bulbs (4.62\%), flowers and rhizome (3.08\%; each) and whole plant (1.54\%) (Figure 2). With regards to the conditions of plants sold, $80.30 \%$ of the parts sold were dried, while $12.12 \%$ and $7.58 \%$ were fresh and powdered form respectively. The contribution of the plant parts sold, and their state (dried, fresh, powdered) is presented on a flow diagram in Figure 3. The mode of preparation of remedies decoction mode was the most common, with oral administration being the most common means of taking remedies. 
Ethnobotany Research and Applications

Table 1. Plant species traded in the Ho Central Market with their ethnobotanical indices based on vendors' knowledge of their application

\begin{tabular}{|c|c|c|c|c|c|c|c|c|c|c|c|c|}
\hline Family & Scientific name & Local names & $\begin{array}{l}\text { Voucher } \\
\text { ID }\end{array}$ & Habit & Status & $\begin{array}{l}\text { Conservation } \\
\text { status }\end{array}$ & URs & FC & NU & $\mathbf{C l}$ & RFC & $\mathbf{R I}$ \\
\hline Amaryllidaceae & Allium cepa L. & Sabala ga & $\begin{array}{l}\text { UHAS/ITA } \\
\text { M/2021/B } \\
\text { B001 }\end{array}$ & Herb & Cultivated & Not Listed & 3 & 1 & 3 & 0.136 & 0.045 & 0.238 \\
\hline Amaryllidaceae & Allium sativum $\mathrm{L}$. & Ayo & $\begin{array}{l}\text { UHAS/ITA } \\
\text { M/2021/B } \\
\text { B002 }\end{array}$ & Herb & Cultivated & Least Concern & 2 & 1 & 2 & 0.091 & 0.045 & 0.175 \\
\hline Anacardiaceae & Mangifera indica $\mathrm{L}$. & Mango & $\begin{array}{l}\text { UHAS/ITA } \\
\text { M/2021/S } \\
\text { B009 }\end{array}$ & Tree & $\begin{array}{l}\text { Wild/ } \\
\text { Cultivated }\end{array}$ & Data Deficient & 4 & 1 & 4 & 0.182 & 0.045 & 0.300 \\
\hline Annonaceae & $\begin{array}{l}\text { Monodora myristica } \\
\text { (Gaertn.) Dunal }\end{array}$ & Ayiku; Yikwi & $\begin{array}{l}\text { UHAS/ITA } \\
\text { M/2021/F } \\
\text { R015 }\end{array}$ & Tree & $\begin{array}{l}\text { Wild/ } \\
\text { Cultivated }\end{array}$ & Least Concern & 6 & 2 & 3 & 0.273 & 0.091 & 0.288 \\
\hline Annonaceae & $\begin{array}{l}\text { Xylopia aethiopica (Dunal) } \\
\text { A.Rich. }\end{array}$ & Etso; Tso & $\begin{array}{l}\text { UHAS/ITA } \\
\text { M/2021/F } \\
\text { R24 }\end{array}$ & Tree & $\begin{array}{l}\text { Wild/ } \\
\text { Cultivated }\end{array}$ & Least Concern & 12 & 6 & 3 & 0.545 & 0.273 & 0.488 \\
\hline Apiaceae & Cuminum cyminum $\mathrm{L}$. & Ahaliwoe & $\begin{array}{l}\text { UHAS/ITA } \\
\text { M/2021/F } \\
\text { R010 }\end{array}$ & Herb & Cultivated & Not Listed & 4 & 2 & 2 & 0.182 & 0.091 & 0.225 \\
\hline Apiaceae & Pimpinella anisum $\mathrm{L}$. & Anise & $\begin{array}{l}\text { UHAS/ITA } \\
\text { M/2021/F } \\
\text { R017 }\end{array}$ & Shrub & $\begin{array}{l}\text { Wild/ } \\
\text { Cultivated }\end{array}$ & Not Listed & 6 & 2 & 6 & 0.273 & 0.091 & 0.475 \\
\hline Apocynaceae & Alstonia boonei De Wild. & Siaketekre & $\begin{array}{l}\text { UHAS/ITA } \\
\text { M/2021/S } \\
\text { B004 }\end{array}$ & Tree & Wild & Least Concern & 1 & 1 & 1 & 0.045 & 0.045 & 0.112 \\
\hline Apocynaceae & $\begin{array}{l}\text { Mondia whitei (Hook.f.) } \\
\text { Skeels }\end{array}$ & Kedeke & $\begin{array}{l}\text { UHAS/ITA } \\
\text { M/2021/R } \\
005\end{array}$ & Herb & Wild & Not Listed & 4 & 4 & 1 & 0.182 & 0.182 & 0.262 \\
\hline Apocynaceae & $\begin{array}{l}\text { Picralima nitida (Stapf) T. } \\
\text { Durand \& H. Durand }\end{array}$ & Quinine ku & $\begin{array}{l}\text { UHAS/ITA } \\
\text { M/2021/F } \\
\text { R016 }\end{array}$ & Tree & Wild & Not Listed & 17 & 4 & 5 & 0.773 & 0.182 & 0.512 \\
\hline
\end{tabular}


Ethnobotany Research and Applications

\begin{tabular}{|c|c|c|c|c|c|c|c|c|c|c|c|c|}
\hline Apocynaceae & $\begin{array}{l}\text { Voacanga africana Stapf. } \\
\text { ex Scott-Elliot }\end{array}$ & Foba & $\begin{array}{l}\text { UHAS/ITA } \\
\text { M/2021/F } \\
\text { R023 }\end{array}$ & Tree & $\begin{array}{l}\text { Wild/ } \\
\text { Cultivated }\end{array}$ & Not Listed & 6 & 2 & 3 & 0.273 & 0.091 & 0.288 \\
\hline Araliaceae & $\begin{array}{l}\text { Pseudopanax arboreus } \\
\text { (L.f.) K.Koch }\end{array}$ & Asikuiator & $\begin{array}{l}\text { UHAS/ITA } \\
\text { M/2021/S } \\
\text { B010 }\end{array}$ & Tree & $\begin{array}{l}\text { Wild/ } \\
\text { Cultivated }\end{array}$ & Not Listed & 2 & 1 & 2 & 0.091 & 0.045 & 0.175 \\
\hline Arecaceae & Cocos nucifera L. & Agone & $\begin{array}{l}\text { UHAS/ITA } \\
\text { M/2021/F } \\
\text { R008 }\end{array}$ & Tree & Cultivated & Not Listed & 1 & 1 & 1 & 0.045 & 0.045 & 0.112 \\
\hline Asparagaceae & $\begin{array}{l}\text { Drimia numidica (Jord. \& } \\
\text { Fourr.) J.C.Manning \& } \\
\text { Goldblatt }\end{array}$ & Doli sabala & $\begin{array}{l}\text { UHAS/ITA } \\
\text { M/2021/B } \\
\text { B003 }\end{array}$ & Shrub & Wild & Not Listed & 1 & 1 & 1 & 0.045 & 0.045 & 0.112 \\
\hline Asteraceae & $\begin{array}{l}\text { Ageratum conyzoides (L.) } \\
\text { L. }\end{array}$ & Efoe & $\begin{array}{l}\text { UHAS/ITA } \\
\mathrm{M} / 2021 / \mathrm{R} \\
002\end{array}$ & Herb & Wild & Least Concern & 3 & 1 & 3 & 0.136 & 0.045 & 0.238 \\
\hline Balanophoraceae & $\begin{array}{l}\text { Thonningia sanguinea } \\
\text { Vahl. }\end{array}$ & Anyigbade & $\begin{array}{l}\text { UHAS/ITA } \\
\text { M/2021/W } \\
\text { P001 }\end{array}$ & Herb & Wild & Not Listed & 12 & 5 & 4 & 0.545 & 0.227 & 0.500 \\
\hline Bignoniaceae & $\begin{array}{l}\text { Kigelia africana (Lam.) } \\
\text { Beneth. }\end{array}$ & Nyakpekpe & $\begin{array}{l}\text { UHAS/ITA } \\
\text { M/2021/F } \\
\text { R013 }\end{array}$ & Tree & $\begin{array}{l}\text { Wild/ } \\
\text { Cultivated }\end{array}$ & Least Concern & 11 & 3 & 7 & 0.500 & 0.136 & 0.588 \\
\hline Brassicaceae & $\begin{array}{l}\text { Anastatica hierochuntica } \\
\text { L. }\end{array}$ & Jericho rose & $\begin{array}{l}\text { UHAS/ITA } \\
\text { M/2021/A } \\
\text { P001 }\end{array}$ & Herb & Wild & Not Listed & 2 & 1 & 2 & 0.091 & 0.045 & 0.175 \\
\hline Clusiaceae & Garcinia kola Heckel & Yikui & $\begin{array}{l}\text { UHAS/ITA } \\
\text { M/2021/F } \\
\text { R012 } \\
\end{array}$ & Tree & $\begin{array}{l}\text { Wild/ } \\
\text { Cultivated }\end{array}$ & Vulnerable & 2 & 1 & 2 & 0.091 & 0.045 & 0.175 \\
\hline Combretaceae & $\begin{array}{l}\text { Pteleopsis suberosa Engl. } \\
\text { \& Diels }\end{array}$ & Gbagaluwa & $\begin{array}{l}\text { UHAS/ITA } \\
\text { M/2021/S } \\
\text { B011 }\end{array}$ & Tree & Wild & Not Listed & 12 & 6 & 2 & 0.545 & 0.273 & 0.425 \\
\hline Compositae & $\begin{array}{l}\text { Tithonia diversifolia } \\
\text { (Hemsl.) A.Gray }\end{array}$ & Agbale & $\begin{array}{l}\text { UHAS/ITA } \\
\mathrm{M} / 2021 / \mathrm{R} \\
009 \\
\end{array}$ & Herb & $\begin{array}{l}\text { Wild/ } \\
\text { Cultivated }\end{array}$ & Not Listed & 1 & 1 & 1 & 0.045 & 0.045 & 0.112 \\
\hline Cucurbitaceae & $\begin{array}{l}\text { Cucumeropsis mannii } \\
\text { Naudin }\end{array}$ & Guzi & $\begin{array}{l}\text { UHAS/ITA } \\
\text { M/2021/S } \\
004\end{array}$ & $\begin{array}{l}\text { Climbing } \\
\text { herb }\end{array}$ & Cultivated & Not Listed & 1 & 1 & 1 & 0.045 & 0.045 & 0.112 \\
\hline
\end{tabular}


Ethnobotany Research and Applications

\begin{tabular}{|c|c|c|c|c|c|c|c|c|c|c|c|c|}
\hline Cucurbitaceae & $\begin{array}{l}\text { Lagenaria siceraria } \\
\text { (Molina) Standl. }\end{array}$ & Ego; Goe & $\begin{array}{l}\text { UHAS/ITA } \\
\text { M/2021/F } \\
\text { R014 }\end{array}$ & Herb & Wild & Not Listed & 3 & 1 & 3 & 0.136 & 0.045 & 0.238 \\
\hline Cucurbitaceae & Momordica charantia L. & Kakle & $\begin{array}{l}\text { UHAS/ITA } \\
\text { M/2021/A } \\
\text { P003 }\end{array}$ & $\begin{array}{l}\text { Climbing } \\
\text { herb }\end{array}$ & Wild & Least Concern & 5 & 1 & 5 & 0.227 & 0.045 & 0.362 \\
\hline Euphorbiaceae & Euphorbia hirta L. & Notsigbe & $\begin{array}{l}\text { UHAS/ITA } \\
\text { M/2021/A } \\
\text { P002 }\end{array}$ & Herb & Wild & Not Listed & 2 & 1 & 2 & 0.091 & 0.045 & 0.175 \\
\hline Euphorbiaceae & Ricinus communis $\mathrm{L}$. & $\begin{array}{l}\text { Gbale; } \\
\text { Dzongbale }\end{array}$ & $\begin{array}{l}\text { UHAS/ITA } \\
\text { M/2021/F } \\
\text { R020 }\end{array}$ & Herb & Wild & Not Listed & 3 & 2 & 3 & 0.136 & 0.091 & 0.288 \\
\hline Fabaceae & $\begin{array}{l}\text { Caesalpinia bonduc (L.) } \\
\text { Roxb. }\end{array}$ & $\begin{array}{l}\text { Adiku; Wole } \\
\text { ade }\end{array}$ & $\begin{array}{l}\text { UHAS/ITA } \\
\text { M/2021/R } \\
004\end{array}$ & $\begin{array}{l}\text { Climbing } \\
\text { shrub }\end{array}$ & Wild & Least Concern & 1 & 1 & 1 & 0.045 & 0.045 & 0.112 \\
\hline Fabaceae & Cajanus cajan (L.) Millsp & $\begin{array}{l}\text { Dedekuad; } \\
\text { adekude }\end{array}$ & $\begin{array}{l}\text { UHAS/ITA } \\
\text { M/2021/S } \\
\text { B012 }\end{array}$ & Shrub & Cultivated & Not Listed & 4 & 1 & 4 & 0.182 & 0.045 & 0.300 \\
\hline Fabaceae & Dioclea reflexa Hook. f. & Adi & $\begin{array}{l}\text { UHAS/ITA } \\
\text { M/2021/F } \\
\text { R011 }\end{array}$ & $\begin{array}{l}\text { Climbing } \\
\text { shrub }\end{array}$ & Wild & Not Listed & 3 & 2 & 3 & 0.136 & 0.091 & 0.288 \\
\hline Fabaceae & Mucuna pruriens (L.) DC. & Tsapkoe & $\begin{array}{l}\text { UHAS/ITA } \\
\text { M/2021/S } \\
005\end{array}$ & $\begin{array}{l}\text { Climbing } \\
\text { shrub }\end{array}$ & $\begin{array}{l}\text { Wild/ } \\
\text { Cultivated }\end{array}$ & Least Concern & 4 & 2 & 2 & 0.182 & 0.091 & 0.225 \\
\hline Fabaceae & $\begin{array}{l}\text { Tetrapleura tetraptera } \\
\text { (Schum and Thonn) Taub. }\end{array}$ & Prekese & $\begin{array}{l}\text { UHAS/ITA } \\
\text { M/2021/F } \\
\text { R022 }\end{array}$ & Tree & $\begin{array}{l}\text { Wild/ } \\
\text { Cultivated }\end{array}$ & Least Concern & 11 & 6 & 5 & 0.500 & 0.273 & 0.613 \\
\hline Gentianaceae & $\begin{array}{l}\text { Anthocleista djalonensis } \\
\text { A. Chev. }\end{array}$ & Gboloba & $\begin{array}{l}\text { UHAS/ITA } \\
\text { M/2021/R } \\
003 \\
\end{array}$ & Tree & Wild & Least Concern & 1 & 1 & 1 & 0.045 & 0.045 & 0.112 \\
\hline Lamiaceae & $\begin{array}{l}\text { Clerodendrum capitatum } \\
\text { (Willd.) Schumach. \& } \\
\text { Thonn. }\end{array}$ & Ayeti & $\begin{array}{l}\text { UHAS/ITA } \\
\text { M/2021/S } \\
\text { B006 }\end{array}$ & Shrub & Wild & Least Concern & 4 & 1 & 4 & 0.182 & 0.045 & 0.300 \\
\hline Lamiaceae & Ocimum basilicum $\mathrm{L}$. & Ahame & $\begin{array}{l}\text { UHAS/ITA } \\
\text { M/2021/A } \\
\text { P004 }\end{array}$ & Herb & $\begin{array}{l}\text { Wild/ } \\
\text { Cultivated }\end{array}$ & Not Listed & 1 & 1 & 1 & 0.045 & 0.045 & 0.112 \\
\hline
\end{tabular}


Ethnobotany Research and Applications

\begin{tabular}{|c|c|c|c|c|c|c|c|c|c|c|c|c|}
\hline Lauraceae & $\begin{array}{l}\text { Cinnamomum zeylanicum } \\
\text { Blume }\end{array}$ & Ami veveti & $\begin{array}{l}\text { UHAS/ITA } \\
\text { M/2021/S } \\
\text { B005 }\end{array}$ & Tree & $\begin{array}{l}\text { Wild/ } \\
\text { Cultivated }\end{array}$ & Least Concern & 9 & 3 & 8 & 0.409 & 0.136 & 0.650 \\
\hline Malvaceae & Adansonia digitata $\mathrm{L}$. & $\begin{array}{l}\text { Adewudati; } \\
\text { Adido }\end{array}$ & $\begin{array}{l}\text { UHAS/ITA } \\
\text { M/2021/S } \\
\text { B003 }\end{array}$ & Tree & Wild & Not Listed & 12 & 10 & 4 & 0.545 & 0.455 & 0.750 \\
\hline Malvaceae & $\begin{array}{l}\text { Cola nitida (Vent.) Schott } \\
\& \text { Endl. }\end{array}$ & Bisi & $\begin{array}{l}\text { UHAS/ITA } \\
\text { M/2021/F } \\
\text { R009 }\end{array}$ & Tree & Wild & Least Concern & 8 & 4 & 4 & 0.364 & 0.182 & 0.450 \\
\hline Malvaceae & Corchorus olitorius L. & Ademe & $\begin{array}{l}\text { UHAS/ITA } \\
\text { M/2021/L0 } \\
07\end{array}$ & Herb & Cultivated & Not Listed & 1 & 1 & 1 & 0.045 & 0.045 & 0.112 \\
\hline Malvaceae & Hibiscus sabdariffa L. & Evema & $\begin{array}{l}\text { UHAS/ITA } \\
\text { M/2021/FL } \\
003\end{array}$ & Shrub & Cultivated & Not Listed & 13 & 7 & 2 & 0.591 & 0.318 & 0.475 \\
\hline Marantaceae & $\begin{array}{l}\text { Thaumatococcus daniellii } \\
\text { (Benn.) Benth. }\end{array}$ & Adaa & $\begin{array}{l}\text { UHAS/ITA } \\
\text { M/2021/L0 } \\
12\end{array}$ & Herb & Wild & Not Listed & 18 & 9 & 4 & 0.818 & 0.409 & 0.700 \\
\hline Meliaceae & $\begin{array}{l}\text { Khaya senegalensis (Desr.) } \\
\text { A.Juss. }\end{array}$ & Logo & $\begin{array}{l}\text { UHAS/ITA } \\
\text { M/2021/S } \\
\text { B007 }\end{array}$ & Tree & $\begin{array}{l}\text { Wild/ } \\
\text { Cultivated }\end{array}$ & Vulnerable & 16 & 6 & 4 & 0.727 & 0.273 & 0.550 \\
\hline Menispermaceae & $\begin{array}{l}\text { Sphenocentrum jollyanum } \\
\text { Pierre }\end{array}$ & Kraman kote & $\begin{array}{l}\text { UHAS/ITA } \\
\text { M/2021/R } \\
008\end{array}$ & $\begin{array}{l}\text { Climbing } \\
\text { herb }\end{array}$ & $\begin{array}{l}\text { Wild/ } \\
\text { Cultivated }\end{array}$ & Least Concern & 4 & 1 & 4 & 0.182 & 0.045 & 0.300 \\
\hline Moringaceae & Moringa oleifera Lam. & $\begin{array}{l}\text { Babatsi; } \\
\text { Yevutsi }\end{array}$ & $\begin{array}{l}\text { UHAS/ITA } \\
\text { M/2021/L0 } \\
11\end{array}$ & Tree & $\begin{array}{l}\text { Wild/ } \\
\text { Cultivated }\end{array}$ & Least Concern & 2 & 1 & 2 & 0.091 & 0.045 & 0.175 \\
\hline Myrtaceae & $\begin{array}{l}\text { Syzygium aromaticum (L.) } \\
\text { Merr. \& L.M.Perry }\end{array}$ & Kpetonku & $\begin{array}{l}\text { UHAS/ITA } \\
\text { M/2021/F } \\
\text { R021 }\end{array}$ & Tree & $\begin{array}{l}\text { Wild/ } \\
\text { Cultivated }\end{array}$ & Not Listed & 11 & 5 & 6 & 0.500 & 0.227 & 0.625 \\
\hline Phyllanthaceae & $\begin{array}{l}\text { Phyllanthus fraternus } \\
\text { G.L.Webster }\end{array}$ & $\begin{array}{l}\text { Kokloblikui; } \\
\text { Lume }\end{array}$ & $\begin{array}{l}\text { UHAS/ITA } \\
\text { M/2021/A } \\
\text { P005 }\end{array}$ & Herb & Wild & Not Listed & 9 & 2 & 6 & 0.409 & 0.091 & 0.475 \\
\hline Piperaceae & $\begin{array}{l}\text { Piper guineense } \\
\text { Schumach. \& Thonn. }\end{array}$ & $\begin{array}{l}\text { Kaleke; } \\
\text { Kukuabe }\end{array}$ & $\begin{array}{l}\text { UHAS/ITA } \\
\text { M/2021/F } \\
\text { R018 }\end{array}$ & $\begin{array}{l}\text { Climbing } \\
\text { herb }\end{array}$ & $\begin{array}{l}\text { Wild/ } \\
\text { Cultivated }\end{array}$ & Least Concern & 8 & 3 & 4 & 0.364 & 0.136 & 0.400 \\
\hline
\end{tabular}


Ethnobotany Research and Applications

\begin{tabular}{|c|c|c|c|c|c|c|c|c|c|c|c|c|}
\hline Plantaginaceae & Plantago ovata Forssk. & Psyllium & $\begin{array}{l}\text { UHAS/ITA } \\
\text { M/2021/F } \\
\text { R019 }\end{array}$ & Herb & $\begin{array}{l}\text { Wild/ } \\
\text { Cultivated }\end{array}$ & Not Listed & 2 & 2 & 1 & 0.091 & 0.091 & 0.162 \\
\hline Poaceae & $\begin{array}{l}\text { Cymbopogon citratus } \\
\text { (DC.) Stapf }\end{array}$ & Tigbe & $\begin{array}{l}\text { UHAS/ITA } \\
\text { M/2021/L0 } \\
09\end{array}$ & Herb & Cultivated & Not Listed & 9 & 3 & 3 & 0.409 & 0.136 & 0.338 \\
\hline Polygalaceae & $\begin{array}{l}\text { Securidaca } \\
\text { longepedunculata Fresen. }\end{array}$ & $\begin{array}{l}\text { Kyirituo; } \\
\text { egbéwoyé }\end{array}$ & $\begin{array}{l}\text { UHAS/ITA } \\
\text { M/2021/S } \\
\text { B013 }\end{array}$ & Herb & Wild & Least Concern & 6 & 2 & 3 & 0.273 & 0.091 & 0.288 \\
\hline Rubiaceae & Morinda lucida Benth. & Ake; Dadklan & $\begin{array}{l}\text { UHAS/ITA } \\
\text { M/2021/R } \\
006\end{array}$ & Tree & Wild & Least Concern & 3 & 2 & 2 & 0.136 & 0.091 & 0.225 \\
\hline Rubiaceae & Nauclea latifolia Sm. & Nyimo & $\begin{array}{l}\text { UHAS/ITA } \\
\text { M/2021/R } \\
007\end{array}$ & Tree & Wild & Least Concern & 7 & 4 & 3 & 0.318 & 0.182 & 0.388 \\
\hline Rutaceae & $\begin{array}{l}\text { Citrus aurantiifolia } \\
\text { (Christm.) Swingle }\end{array}$ & $\begin{array}{l}\text { Mumoe; } \\
\text { Donuti }\end{array}$ & $\begin{array}{l}\text { UHAS/ITA } \\
\text { M/2021/F } \\
\text { R007 }\end{array}$ & Tree & $\begin{array}{l}\text { Wild/ } \\
\text { Cultivated }\end{array}$ & Not Listed & 4 & 2 & 3 & 0.182 & 0.091 & 0.288 \\
\hline Rutaceae & $\begin{array}{l}\text { Zanthoxylum } \\
\text { zanthoxyloides L. }\end{array}$ & Exe & $\begin{array}{l}\text { UHAS/ITA } \\
\text { M/2021/R } \\
010\end{array}$ & Tree & Wild & Least Concern & 23 & 9 & 7 & 1.045 & 0.409 & 0.887 \\
\hline Sapotaceae & $\begin{array}{l}\text { Butyrospermum parkii } \\
\text { (G.Don) Kotschy }\end{array}$ & Yo & $\begin{array}{l}\text { UHAS/ITA } \\
\text { M/2021/F } \\
\text { R005 }\end{array}$ & Tree & Wild & Not Listed & 2 & 1 & 2 & 0.091 & 0.045 & 0.175 \\
\hline Solanaceae & Capsicum frutescens $\mathrm{L}$. & Atadi & $\begin{array}{l}\text { UHAS/ITA } \\
\text { M/2021/F } \\
\text { R006 }\end{array}$ & Shrub & $\begin{array}{l}\text { Wild/ } \\
\text { Cultivated }\end{array}$ & Least Concern & 7 & 6 & 2 & 0.318 & 0.273 & 0.425 \\
\hline Solanaceae & Solanum macrocarpon L. & Gboma & $\begin{array}{l}\text { UHAS/ITA } \\
\text { M/2021/L0 } \\
08\end{array}$ & Herb & $\begin{array}{l}\text { Wild/ } \\
\text { Cultivated }\end{array}$ & Not Listed & 2 & 1 & 2 & 0.091 & 0.045 & 0.175 \\
\hline Verbenaceae & $\begin{array}{l}\text { Lippia multiflora } \\
\text { Moldenke }\end{array}$ & Avudati & $\begin{array}{l}\text { UHAS/ITA } \\
\text { M/2021/L0 } \\
10\end{array}$ & Shrub & Wild & Not Listed & 3 & 2 & 2 & 0.136 & 0.091 & 0.225 \\
\hline Zingiberaceae & $\begin{array}{l}\text { Aframomum melegueta K. } \\
\text { Schum. }\end{array}$ & $\begin{array}{l}\text { Awusa; } \\
\text { Atakui }\end{array}$ & $\begin{array}{l}\text { UHAS/ITA } \\
\text { M/2021/S } \\
003\end{array}$ & Herb & Wild & Data Deficient & 12 & 5 & 4 & 0.545 & 0.227 & 0.500 \\
\hline
\end{tabular}




\begin{tabular}{|c|c|c|c|c|c|c|c|c|c|c|c|c|}
\hline Zingiberaceae & Curcuma longa $\mathrm{L}$. & Tumeric & $\begin{array}{l}\text { UHAS/ITA } \\
\text { M/2021/R } \\
\text { H001 }\end{array}$ & Herb & Cultivated & Data Deficient & 1 & 1 & 1 & 0.045 & 0.045 & 0.112 \\
\hline Zingiberaceae & Zingiber officinale Roscoe. & Agumetaku & $\begin{array}{l}\text { UHAS/ITA } \\
\text { M/2021/R } \\
\mathrm{H} 002\end{array}$ & Herb & $\begin{array}{l}\text { Wild/ } \\
\text { Cultivated }\end{array}$ & Data Deficient & 5 & 3 & 4 & 0.227 & 0.136 & 0.400 \\
\hline
\end{tabular}

Use report (UR), Cultural importance (CI), Frequency of citation ( $\mathrm{FC})$, Number of uses (NU), Relative frequency of citation (RFC), Relative importance index (RI) 


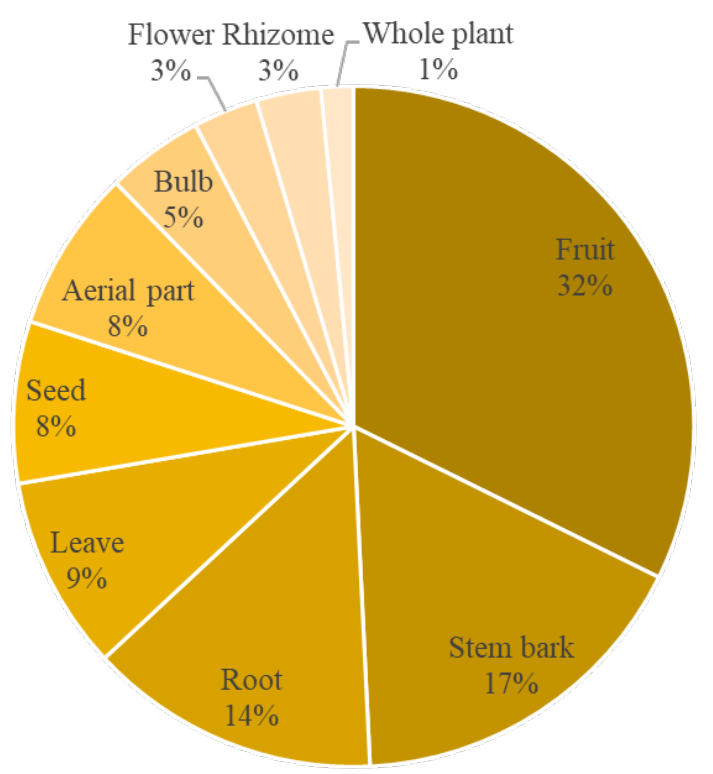

Figure 2. Plant parts sold in the Ho central market as percentage of the total number of species $(n=60)$

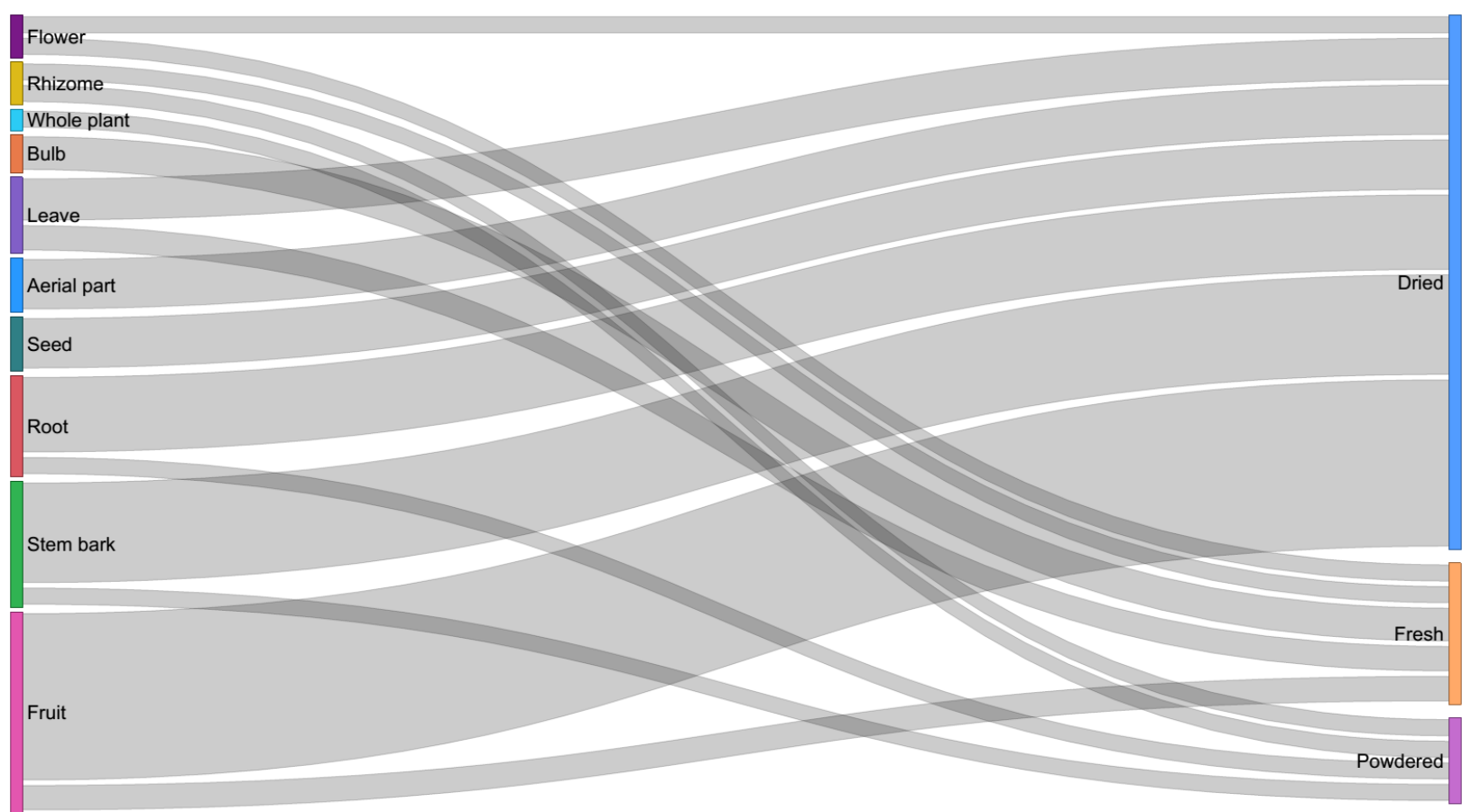

Figure 3. The flow connections between plant parts sold and their condition

\section{Quantitative ethnobotanical indices for commercialized plants}

The highest FC value ( $\mathrm{n}=10$ ) was recorded for Adansonia digitata, followed by Thaumatococcus daniellii and Zanthoxylum zanthoxyloides $(\mathrm{n}=9$; each) and Hibiscus sabdariffa $(\mathrm{n}=7)$, thus were sold by 10,9 , and 7 out of the 22 vendors respectively. The FC value was the same for $K$. senegalensis, Xylopia aethiopica, Pteleopsis suberosa, Tetrapleura tetraptera and Capsicum frutescens $(\mathrm{n}=6)$, while Thonningia sanguinea, Aframomum melegueta, and Syzygium aromaticum had FC $(\mathrm{n}=5)$.

With regard to use reports (UR), Z. zanthoxyloides had the highest UR value $(\mathrm{n}=23)$ that was followed by $T$. daniellii $(\mathrm{n}=18)$, Picralima nitida $(\mathrm{n}=17), K$. senegalensis $(\mathrm{n}=16)$, and H. sabdariffa $(\mathrm{n}=13)$. The $X$. aethiopica, T. sanguinea, $P$. suberosa, $A$. digitata and $A$. melegueta had the same UR value ( $\mathrm{n}=12$; each) while Kigelia africana, T. tetraptera, and $S$. aromaticum had the same UR value $(n=11$; each). The cultural importance $(\mathrm{Cl})$ of the species followed the 
same trend as the UR value with $Z$. zanthoxyloides having the highest $\mathrm{CI}$ value (1.045) followed by $T$. daniellii (0.818), $P$. nitida (0.773), K. senegalensis (0.727) and $H$. sabdariffa (0.591). The number of uses (NU) for each species for the different categories of application was highest for C. zeylanicum $(\mathrm{n}=8), K$. africana and $Z$. zanthoxyloides $(\mathrm{n}=7$; each), S. aromaticum, P. fraternus and $P$. anisum ( $\mathrm{n}=6$; each).

With regards to the relative frequency citation (RFC) $A$. digitata had the highest RFC value (0.455), while $T$. daniellii and $Z$. zanthoxyloides had the same RFC (0.409; each) and $H$. sabdariffa $(0.318)$. Unlike the RFC, the relative importance index (RI) was recorded the highest for $Z$. zanthoxyloides (0.887). A. digitata recorded the secondhighest RI value (0.75), followed by T. daniellii $(0.700)$, C. zeylanicum $(0.65)$, S. aromaticum $(0.625)$, and T. tetraptera (0.613).

\section{Plant applications}

A total of 51 applications were recorded for the 60 plant species. The most salient application for the plants based on the use report of vendors familiarization were for body pains $(n=51)$, blood tonic $(n=29)$, abdominal pains ( $n$ $=22)$, immune booster $(n=20)$, sexual weakness, piles, and malaria $(n=19$; each), stomach ulcer $(n=13)$ and as a preservative for traditional medicine $(n=12)$ (Figure 4). The least mentioned ailments include sperm production, prostate disease, kidney disease, and measles ( $n=1$; each).

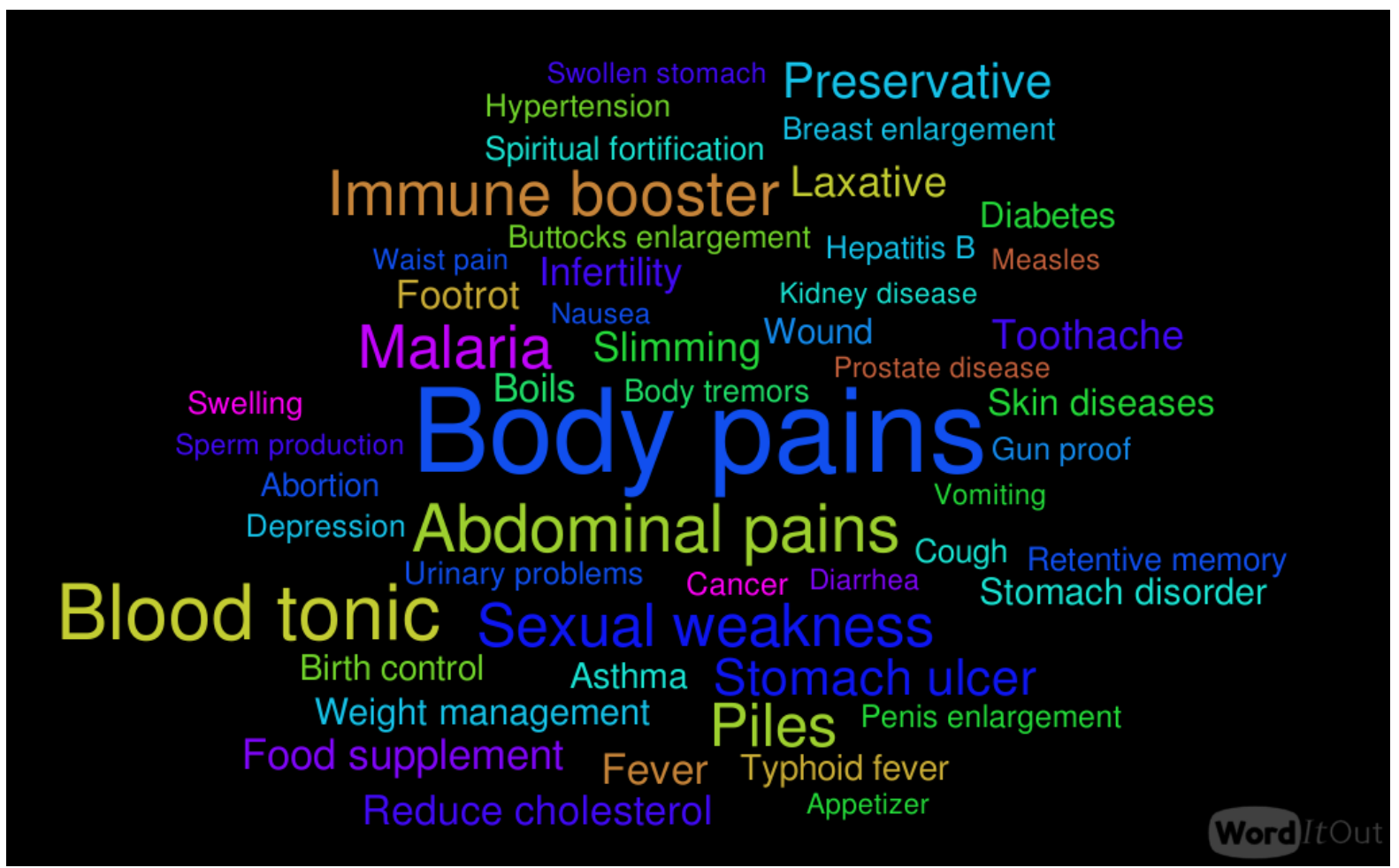

Figure 4. Word cloud of the applications mentioned by vendors for the medicinal plants traded in the Ho Central Market. Maximum mentions are 51 for body pains.

The species used in treating particular ailments is presented in Table 2. The highest number of plant species $(n=$ 22) were used to treat body pains. Based on fidelity level (FL), the most important species for treating body pain were L. siceraria, A. melegueta, P. guineense, C. frutescens, C. cajan, P. arboreus, A. conyzoides, P. suberosa, C. capitatum, $X$. aethiopica and $S$. aromaticum. For the treatment of anemia (blood tonic), the most important species were G. kola, H. sabdariffa, A. hierochuntica, T. daniellii based on fidelity level (Table 2). 
Table 2. Medicinal applications, formulation, route of administration and fidelity level (FL) of plants traded in the Ho Central Market

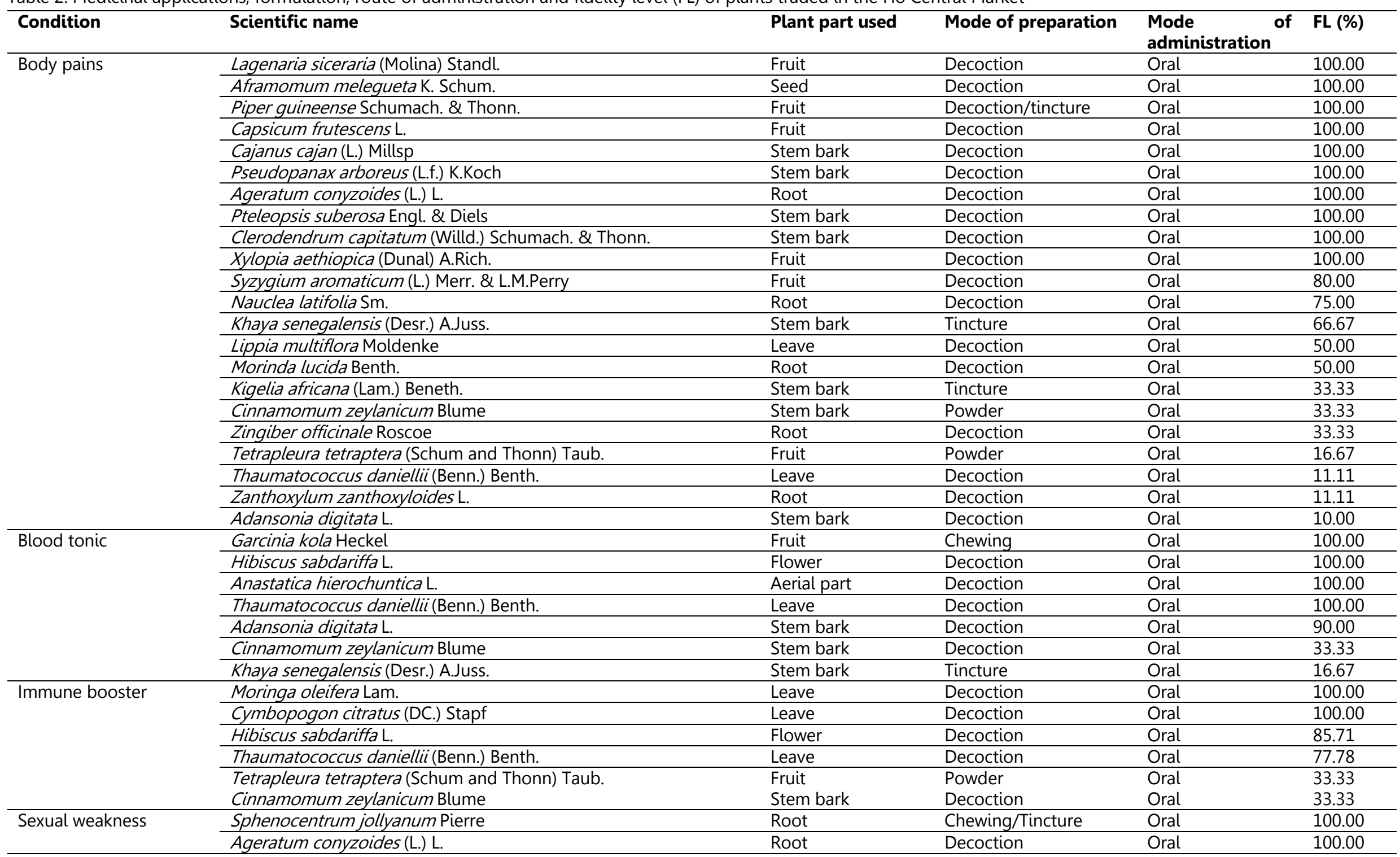




\begin{tabular}{|c|c|c|c|c|c|}
\hline & Garcinia kola Heckel & Fruit & Chewing & Oral & 100.00 \\
\hline & Caesalpinia bonduc (L.) Roxb. & Root & Tincture & Oral & 100.00 \\
\hline & Thonningia sanguinea Vahl & Root & Decoction & Oral & 100.00 \\
\hline & Mondia whitei (Hook.f.) Skeels & Root & Chewing & Oral & 100.00 \\
\hline & Monodora myristica (Gaertn.) Dunal & Fruit & Decoction & Oral & 100.00 \\
\hline & Tithonia diversifolia (Hemsl.) A.Gray & Root & Decoction & Oral & 100.00 \\
\hline & Ricinus communis $\mathrm{L}$. & Fruit & Decoction & Oral & 50.00 \\
\hline & Kigelia africana (Lam.) Beneth. & Stem bark & Tincture & Oral & 33.33 \\
\hline & Thaumatococcus daniellii (Benn.) Benth. & Leave & Decoction & Oral & 11.11 \\
\hline \multirow[t]{6}{*}{ Piles } & Thonningia sanguinea Vahl & Whole plant & Decoction & Oral & 100.00 \\
\hline & Khaya senegalensis (Desr.) A.Juss. & Stem bark & Tincture & Oral & 83.33 \\
\hline & Xylopia aethiopica (Dunal) A.Rich. & Fruit & Decoction & Oral & 83.33 \\
\hline & Piper guineense Schumach. \& Thonn. & Fruit & Decoction & Oral & 66.67 \\
\hline & Cinnamomum zeylanicum Blume & Stem bark & Decoction & Oral & 33.33 \\
\hline & Zanthoxylum zanthoxyloides L. & Root & Decoction & Oral & 11.11 \\
\hline \multirow[t]{8}{*}{ Malaria } & Picralima nitida (Stapf) T. Durand \& H. Durand & Seed & Infusion & Oral & 100.00 \\
\hline & Momordica charantia L. & Aerial parts & Decoction & Oral & 100.00 \\
\hline & Lippia multiflora Moldenke & Leave & Decoction & Oral & 100.00 \\
\hline & Khaya senegalensis (Desr.) A.Juss. & Stem bark & Decoction & Oral & 100.00 \\
\hline & Alstonia boonei De Wild. & Stem bark & Decoction & Oral & 100.00 \\
\hline & Cymbopogon citratus (DC.) Stapf & Flower & Decoction & Oral & 100.00 \\
\hline & Phyllanthus fraternus G.L.Webster & Aerial part & Decoction & Oral & 50.00 \\
\hline & Zanthoxylum zanthoxyloides L. & Root & Decoction & Oral & 11.11 \\
\hline \multirow[t]{5}{*}{ Stomach ulcer } & Picralima nitida (Stapf) T. Durand \& H. Durand & Seed & Decoction & Oral & 100.00 \\
\hline & Cajanus cajan (L.) Millsp & Stem bark & Decoction & Oral & 100.00 \\
\hline & Pteleopsis suberosa Engl. \& Diels & Stem bark & Decoction & Oral & 100.00 \\
\hline & Clerodendrum capitatum (Willd.) Schumach. \& Thonn. & Stem bark & Decoction & Oral & 100.00 \\
\hline & Pimpinella anisum $\mathrm{L}$. & Fruit & Decoction & Oral & 50.00 \\
\hline \multirow[t]{5}{*}{ Preservative } & Aframomum melegueta K. Schum. & Seed & Decoction & & 100.00 \\
\hline & Monodora myristica (Gaertn.) Dunal & Fruit & Tincture & & 100.00 \\
\hline & Syzygium aromaticum (L.) Merr. \& L.M.Perry & Fruit & Decoction & & 60.00 \\
\hline & Citrus aurantiifolia (Christm.) Swingle & Fruit & Decoction & & 50.00 \\
\hline & Xylopia aethiopica (Dunal) A.Rich. & Fruit & Decoction & & 16.67 \\
\hline \multirow[t]{7}{*}{ Abdominal pains } & Zanthoxylum zanthoxyloides L. & Root & Decoction & Oral & 100.00 \\
\hline & Phyllanthus fraternus G.L.Webster & Aerial part & Decoction & Oral & 100.00 \\
\hline & Monodora myristica (Gaertn.) Dunal & Fruit & Tincture & Oral & 100.00 \\
\hline & Mangifera indica $\mathrm{L}$. & Stem bark & Decoction & Oral & 100.00 \\
\hline & Picralima nitida (Stapf) T. Durand \& H. Durand & Seed & Decoction & Oral & 100.00 \\
\hline & Pimpinella anisum $\mathrm{L}$. & Fruit & Infusion & Oral & 50.00 \\
\hline & Nauclea latifolia Sm. & Root & Decoction & Oral & 50.00 \\
\hline
\end{tabular}




\begin{tabular}{|c|c|c|c|c|c|}
\hline & Piper guineense Schumach. \& Thonn. & Fruit & Decoction & Oral & 33.33 \\
\hline & Zingiber officinale Roscoe & Root & Tincture & Oral & 33.33 \\
\hline \multirow[t]{6}{*}{ Fever } & Solanum macrocarpon $\mathrm{L}$. & Leave & Decoction & Oral & 100.00 \\
\hline & Momordica charantia L. & Aerial parts & Decoction & Oral & 100.00 \\
\hline & Phyllanthus fraternus G.L.Webster & Aerial part & Decoction & Oral & 100.00 \\
\hline & Cymbopogon citratus (DC.) Stapf & Flower & Decoction & Oral & 100.00 \\
\hline & Mangifera indica $\mathrm{L}$. & Stem bark & Chewing & Oral & 100.00 \\
\hline & Zanthoxylum zanthoxyloides L. & Root & Decoction & Oral & 11.11 \\
\hline \multirow[t]{6}{*}{ Slimming } & Allium sativum $\mathrm{L}$. & Bulb & Fresh & Oral & 100.00 \\
\hline & Curcuma longa $\mathrm{L}$. & Rhizome & Powder & Oral & 100.00 \\
\hline & Moringa oleifera Lam. & Leave & Decoction & Oral & 100.00 \\
\hline & Cinnamomum zeylanicum Blume & Stem bark & Powder & Oral & 66.67 \\
\hline & Citrus aurantiifolia (Christm.) Swingle & Fruit & Juice & Oral & 50.00 \\
\hline & Tetrapleura tetraptera (Schu \& Thonn) Taub. & Fruit & Decoction & Oral & 33.33 \\
\hline \multirow[t]{6}{*}{ Laxative } & Picralima nitida (Stapf) T. Durand \& H. Durand & Seed & Decoction & Oral & 100.00 \\
\hline & Plantago ovata Forssk. & Fruit & Infusion & Oral & 100.00 \\
\hline & Cucumeropsis mannii Naudin & Seed & Decoction & Oral & 100.00 \\
\hline & Zingiber officinale Roscoe & Root & Tincture & Oral & 33.33 \\
\hline & Capsicum frutescens $\mathrm{L}$. & Fruit & Decoction & Oral & 16.67 \\
\hline & Zanthoxylum zanthoxyloides $\mathrm{L}$. & Root & Decoction & Oral & 11.11 \\
\hline \multirow[t]{5}{*}{ Food supplement } & Corchorus olitorius L. & Leave & Decoction & Oral & 100.00 \\
\hline & Solanum macrocarpon L. & Leave & Decoction & Oral & 100.00 \\
\hline & Mucuna pruriens (L.) DC. & Seed & Decoction & Oral & 100.00 \\
\hline & Cuminum cyminum $\mathrm{L}$. & Fruit & Add to honey & Oral & 100.00 \\
\hline & Adansonia digitata $\mathrm{L}$. & Stem bark & Decoction & Oral & 10.00 \\
\hline Toothache & Zanthoxylum zanthoxyloides L. & Root & Decoction & Oral & 88.89 \\
\hline \multirow[t]{4}{*}{ Reduce cholesterol } & Allium cepa $\mathrm{L}$. & Bulb & Chewing & Oral & 100.00 \\
\hline & Citrus aurantiifolia (Christm.) Swingle & Fruit & Juice & Oral & 100.00 \\
\hline & Allium sativum $\mathrm{L}$. & Bulb & Decoction & Oral & 100.00 \\
\hline & Tetrapleura tetraptera (Schum and Thonn) Taub. & Fruit & Decoction & Oral & 66.67 \\
\hline \multirow[t]{5}{*}{ Foot rot } & Cajanus cajan (L.) Millsp & Stem bark & Decoction & Topical & 100.00 \\
\hline & Clerodendrum capitatum (Willd.) Schumach. \& Thonn. & Stem bark & Decoction & Oral & 100.00 \\
\hline & Cola nitida (Vent.) Schott \& Endl. & Fruit & Decoction & Oral & 50.00 \\
\hline & Pimpinella anisum $\mathrm{L}$. & Fruit & Decoction & Oral & 50.00 \\
\hline & Syzygium aromaticum (L.) Merr. \& L.M.Perry & Fruit & Decoction & Oral & 20.00 \\
\hline \multirow[t]{5}{*}{ Infertility } & Ocimum basilicum $\mathrm{L}$. & Aerial part & Infusion & Oral & 100.00 \\
\hline & Cocos nucifera $\mathrm{L}$. & Fruit & Drink/Chewing & Oral & 100.00 \\
\hline & Anthocleista djalonensis A. Chev. & Root & Decoction & Oral & 100.00 \\
\hline & Nauclea latifolia Sm. & Root & Decoction & Oral & 50.00 \\
\hline & Kigelia africana (Lam.) Beneth. & Stem bark & Decoction & Oral & 33.33 \\
\hline
\end{tabular}


Ethnobotany Research and Applications

\begin{tabular}{|c|c|c|c|c|c|}
\hline \multirow[t]{5}{*}{ Boils } & Allium cepa L. & Bulb & Paste & Topical & 100.00 \\
\hline & Voacanga africana Stapf. ex Scott-Elliot & Fruit & Decoction & Oral & 100.00 \\
\hline & Cinnamomum zeylanicum Blume & Stem bark & Decoction & Oral & 33.33 \\
\hline & Cola nitida (Vent.) Schott \& Endl. & Fruit & Decoction & Oral & 25.00 \\
\hline & Syzygium aromaticum (L.) Merr. \& L.M.Perry & Fruit & Squeeze & Lick/Topical & 20.00 \\
\hline \multirow[t]{4}{*}{ Skin diseases } & Cajanus cajan (L.) Millsp & Stem bark & Decoction & Oral & 100.00 \\
\hline & Voacanga africana Stapf. ex Scott-Elliot & Fruit & Decoction & Oral & 100.00 \\
\hline & Butyrospermum parkii (G.Don) Kotschy & Fruit & Paste & Topical & 100.00 \\
\hline & Clerodendrum capitatum (Willd.) Schumach. \& Thonn. & Stem bark & Decoction & Oral & 100.00 \\
\hline \multirow[t]{3}{*}{ Wound } & Mangifera indica $\mathrm{L}$. & Stem bark & Decoction & Oral & 100.00 \\
\hline & Voacanga africana Stapf. ex Scott-Elliot & Fruit & Decoction & Oral & 100.00 \\
\hline & Butyrospermum parkii (G.Don) Kotschy & Fruit & Paste & Topical & 100.00 \\
\hline \multirow[t]{2}{*}{ Typhoid fever } & Phyllanthus fraternus G.L.Webster & Aerial part & Decoction & Oral & 100.00 \\
\hline & Morinda lucida Benth. & Root & Tincture & Oral & 100.00 \\
\hline \multirow[t]{3}{*}{ Stomach disorder } & Pseudopanax arboreus (L.f.) K.Koch & Stem bark & Decoction & Oral & 100.00 \\
\hline & Piper guineense Schumach. \& Thonn. & Fruit & Decoction & Oral & 66.67 \\
\hline & Syzygium aromaticum (L.) Merr. \& L.M.Perry & Fruit & Decoction & Oral & 20.00 \\
\hline \multirow[t]{3}{*}{ Asthma } & Securidaca longepedunculata Fresen. & Stem bark & Decoction + honey & Oral & 100.00 \\
\hline & Euphorbia hirta L. & Aerial part & Decoction & Oral & 100.00 \\
\hline & Dioclea reflexa (Hook.f) C. Wright & Seed & Decoction & Oral & 50.00 \\
\hline \multirow[t]{4}{*}{ Diabetes } & Allium cepa L. & Bulb & Chewing & Oral & 100.00 \\
\hline & Momordica charantia L. & Aerial parts & Decoction & Oral & 100.00 \\
\hline & Pimpinella anisum $\mathrm{L}$. & Fruit & Decoction & Oral & 50.00 \\
\hline & Adansonia digitata $\mathrm{L}$. & Fruit & Mash & Oral & 10.00 \\
\hline \multirow[t]{2}{*}{ Weight management } & Lagenaria siceraria (Molina) Standl. & Fruit & Decoction & Oral & 100.00 \\
\hline & Cola nitida (Vent.) Schott \& Endl. & Fruit & Chewing & Oral & 100.00 \\
\hline \multirow[t]{4}{*}{ Birth control } & Sphenocentrum jollyanum Pierre & Root & Tincture & Oral & 100.00 \\
\hline & Ricinus communis $\mathrm{L}$. & Fruit & Decoction & Oral & 50.00 \\
\hline & Aframomum melegueta K. Schum. & Seed & Tincture & Oral & 20.00 \\
\hline & Thonningia sanguinea Vahl & Root & Tincture & Oral & 20.00 \\
\hline \multirow[t]{2}{*}{ Retentive memory } & Euphorbia hirta L. & Aerial part & Decoction & Oral & 100.00 \\
\hline & Cuminum cyminum L. & Fruit & Decoction & Oral & 100.00 \\
\hline \multirow[t]{3}{*}{ Cough } & Mangifera indica L. & Stem bark & Decoction & Oral & 100.00 \\
\hline & Pimpinella anisum $\mathrm{L}$. & Fruit & Infusion & Oral & 50.00 \\
\hline & Cola nitida (Vent.) Schott \& Endl. & Fruit & Decoction & Oral & 25.00 \\
\hline \multirow[t]{2}{*}{ Appetizer } & Zingiber officinale Roscoe & Rhizome & Decoction & Oral & 66.67 \\
\hline & Picralima nitida (Stapf) T.Durand \& H.Durand & Seed & Decoction & Oral & 25.00 \\
\hline \multirow[t]{3}{*}{ Abortion } & Sphenocentrum jollyanum Pierre & Root & Decoction & Oral & 100.00 \\
\hline & Aframomum melegueta K. Schum. & Seed & Tincture & Oral & 20.00 \\
\hline & Thonningia sanguinea Vahl & Root & Tincture & Oral & 20.00 \\
\hline
\end{tabular}


Ethnobotany Research and Applications

\begin{tabular}{|c|c|c|c|c|c|}
\hline \multirow[t]{3}{*}{ Spiritual fortification } & Drimia numidica (Jord. \& Fourr.) J.C.Manning \& Goldblatt & Bulb & Infusion & Bathing & 100.00 \\
\hline & Ricinus communis $\mathrm{L}$. & Fruit & Decoction & Oral & 50.00 \\
\hline & Phyllanthus fraternus G.L.Webster & Aerial part & Decoction & Oral & 50.00 \\
\hline Urinary problems & Zanthoxylum zanthoxyloides $\mathrm{L}$. & Root & Decoction & Oral & 22.22 \\
\hline Hypertension & Tetrapleura tetraptera (Schum and Thonn) Taub. & Fruit & Decoction & Oral & 33.33 \\
\hline Body tremors & Mucuna pruriens (L.) DC. & Seed & Decoction & Oral & 100.00 \\
\hline Penis enlargement & Kigelia africana (Lam.) Beneth. & Fruit & Decoction & Oral & 66.67 \\
\hline Breast enlargement & Kigelia africana (Lam.) Beneth. & Fruit & Powder + Shea butter & Topical & 66.67 \\
\hline Buttocks enlargement & Kigelia africana (Lam.) Beneth. & Fruit & Powder + Shea butter & Topical & 66.67 \\
\hline Cancer & Kigelia africana (Lam.) Beneth. & Fruit & Decoction & Oral & 66.67 \\
\hline Swelling & Securidaca longepedunculata Fresen. & Stem bark & Powder + Shea butter & Topical & 100.00 \\
\hline Gun proof & Securidaca longepedunculata Fresen. & Stem bark & Decoction & Oral & 100.00 \\
\hline \multirow[t]{2}{*}{ Hepatitis B } & Momordica charantia L. & Aerial part & Decoction & Oral & 100.00 \\
\hline & Phyllanthus fraternus G.L.Webster & Aerial part & Decoction & Oral & 50.00 \\
\hline \multirow[t]{2}{*}{ Depression } & Lagenaria siceraria (Molina) Standl. & Fruit & Decoction & Oral & 100.00 \\
\hline & Pimpinella anisum $\mathrm{L}$. & Fruit & Decoction & Oral & 50.00 \\
\hline Nausea & Cinnamomum zeylanicum Blume & Stem bark & Decoction & Oral & 33.33 \\
\hline Vomiting & Cinnamomum zeylanicum Blume & Stem bark & Decoction & Oral & 33.33 \\
\hline Diarrhea & Syzygium aromaticum (L.) Merr. \& L.M.Perry & Fruit & Decoction & Oral & 20.00 \\
\hline Waist pain & Sphenocentrum jollyanum Pierre & Root & Tincture & Oral & 100.00 \\
\hline Measles & Momordica charantia $\mathrm{L}$. & Aerial parts & Decoction & Oral & 100.00 \\
\hline Swollen stomach & Anastatica hierochuntica L. & Aerial part & Decoction & Oral & 100.00 \\
\hline Sperm production & Ageratum conyzoides (L.) L. & Root & Decoction & Oral & 100.00 \\
\hline Prostate disease & Dioclea reflexa (Hook.f) C. Wright & Fruit & Powder & Oral & 50.00 \\
\hline Kidney disease & Dioclea reflexa (Hook.f) C. Wright & Fruit & Powder & Oral & 50.00 \\
\hline
\end{tabular}




\section{Discussion}

The use of Fabaceae, Apocynaceae, and Malvaceae families in herbal medicines is documented in Ghana (Asase \& Oppong-Mensah 2009; Boadu \& Asase 2017). The dominance of these three plant families in this study is consistent with other market surveys in the country (Asase \& Oppong-Mensah 2009; van Andel et al. 2012). According to Boadu and Asase (2017), members of these plant families are widely known to contain secondary metabolites such as tannins, phenolics, and alkaloids responsible for their bioactivity. The general knowledge about their bioactivity may account for their dominance in the Ghanaian herbal pharmacopoeia. The dried condition of most plant species traded is consistent with findings from other herbal market surveys in Ghana (Asase \& Oppong-Mensah 2009; Van Andel et al. 2012). The dried plant materials are easy transported and better protected from microorganism infestations as water is the main requirement in microbial growth and thus the primary method used to preserve the plant materials (Asase \& Oppong-Mensah 2009).

The plant species commonly traded in this study differed from market surveys in other places in Ghana. Asase and Oppong-Mensah (2009) found a clear difference in plant species sold for the treatment of malaria to be dissimilar for markets in different political regions in Ghana. Van Andel et al. (2012) also found variation in floristic diversity in different herbal markets across Ghana. According to Thomas et al. (2009) the distribution of knowledge about traditional remedies follows a pattern whereby most knowledge is idiosyncratic while few remedies are known to almost everyone. Idiosyncrasy may account for the variation in medicinal plants sold in different markets. The dynamic nature and non-codified traditional knowledge systems result in variation even for the most prevalent species (Mary et al. 2011). Albuquerque et al. (2007) pointed out that medicinal products traded in markets represent local cultural knowledge and biological diversity. Most species documented in this study are listed in other market surveys. Cultural knowledge about the medicinal application of A. digitata, Z. zanthoxyloides, $T$. daniellii, $P$. nitida, K. senegalensis and $H$. sabdariffa was recorded as idiosyncratic, leading to their high frequency of citation (FC) in this study. Thus, their increased use reports reflect their versatility in the local repertoire of valuable medicinal plant species.

Markets serve as a venue to diffuse empirical knowledge about plants (Bye \& Linares 1983; Albuquerque et al. 2007; Monteiro et al. 2010). Information exchange between vendors, suppliers, and customers usually results in a high level of agreement on the selection of plants and their parts to treat a particular ailment (Albuquerque et al. 2007). The market as an open knowledge system and exchange of information accounted for the high uniformity between the application of the plants mentioned by the vendors and their application by traditional medicine practitioners in Ghana. The use of $P$. guineense and $Z$. zanthoxyloides for the treatment of body pains, M. charantia for malaria and diabetes, $X$. aethiopica and $P$. guineense for piles, A. boonei, C. citratus and $Z$. zanthoxyloides for malaria, $K$. senegalensis for malaria and anemia, $M$. indica for cough, $V$. Africana for wound treatment as well as their mode of preparation and administration are consistent with previous studies (Asase \& Oppong-Mensah 2009; van Andel et al. 2012; Boadu \& Asase 2017; Dogor et al. 2018; Appiah et al. 2019). The traditional medicinal applications of the most culturally important plant species in this study $Z$. zanthoxyloides, was consistent with their application in other countries (Okagu et al. 2021). The findings of the present study is in line with that of Tinitana et al. (2016) and Ouedraogo et al. (2020), which also found a high level of knowledge about the application of medicinal plants among vendors in Ecuador and Burkina Faso, respectively.

Markets typically provide insights into health and illness in society as the trade in medicinal resources reflects the health needs of the local community (Van Andel et al. 2012; Quiroz et al. 2014; Towns et al. 2014; Tinitana et al. 2016; Williams et al. 2000). Culturally important plant species are typically used for a more common ailment (Heinrich et al. 2009). This study revealed that the most culturally significant plants were used to treat the most prevalent diseases in the study area. The most regular prevailing diseases related to pains, hypertension, anemia, malaria, diabetes, asthma, malnutrition and diarrheal are among the top causes of outpatient attendance and deaths in Ghana (University of Ghana 2018; Centre for Disease Control and Prevention-CDC 2019). Accordingly, these indications were amongst the most cited by the vendors and for which the most culturally important species were applied. The most culturally important medicinal plant species are usually linked to local health issues (Bye \& Linares 1983, Cunningham 2001). This study revealed high fidelity levels for the most culturally important species and the most prevalent ailments.

\section{Conclusions}

The findings from this study have revealed that the local market value of 60 plant species were documented. The local market study is an essential source of plant resources for the practice of traditional medicine. The market is a venue for the diffusion of empirical knowledge about plants from different regions and origins for traditional 
medicinal purposes that help maintain local knowledge about medicinal plants. An idiosyncrasy of knowledge due to culture influenced the plant species traded and their use in a community that results in the variation in trade and use for traditional medicinal practice. The market provides an overview of the most prevalent ailments in the community as people become knowledgeable about the culturally bound syndromes in communities.

\section{Declarations}

Ethics approval and consent to participate. All the participants provided prior informed consent before the interviews.

Consent for publication: Not applicable.

Availability of data and materials: Plant materials were prepared and deposited in the herbarium unit of the Institute of Traditional and Alternative Medicine (ITAM), University of Health and Allied Sciences (UHAS).

Competing interests: The authors declared no conflicting interests.

Funding: This research was not supported by any funding from public, private or Non-Governmental Organization (NGO).

Authors' contributions: MKB, AOA, BKT, EDW, MFB and MTB conceived the research idea. MKB and AOA did the data collection. MKB, AOA, EDW and BKT analyzed and interpreted the data. MKB and AOA drafted the initial manuscript, and EDW and MTB revised and improved the manuscript. All the authors read, reviewed, and approved the final version of the manuscript.

\section{Acknowledgement}

The authors would like to thank the traders of traditional medicine products in the Ho Central Market for their contributions to the success of this study. The authors are also grateful to Mathias Gidisu for his assistance with data collection.

\section{Literature cited}

Abbiw DK. 1990. Useful plants of Ghana. Intermediate Technology Publications, London, UK.

Albuquerque UP, Monteiro, JM, Ramos, MA, Amorim, ELC. 2007. Medicinal and magic plants from a public market in northeastern Brazil. Journal of Ethnopharmacology 110(1): 76-91.

Appiah KS, Oppong CP, Mardani HK, Omari RA, Kpabitey S, Amoatey CA, Onwona-Agyeman S, Oikawa Y, Katsura K, Fujii Y. 2019. Medicinal plants used in the Ejisu-Juaben municipality, southern Ghana: An Ethnobotanical Study. Medicines 6(1):1.

Asase L, Oppong-Mensah G. 2009. Traditional antimalarial phytotherapy remedies in herbal markets in southern Ghana. Journal of Ethnopharmacology 126: 492-499.

Boadu AA, Asase A. 2017. Documentation of herbal medicines used for the treatment and management of human diseases by some communities in southern Ghana. Evidence-Based Complementary and Alternative Medicine: eCAM, 3043061

Bussmann RW, Paniagua Zambrana, NY, Romero C, Hart RE. 2018. Astonishing diversity-the medicinal plant markets of Bogotá, Colombia. Journal of Ethnobiology and Ethnomedicine, 14(1), 43.

Bussmann RW, Paniagua Zambrana NY, Moya Huanca, LA, Hart R. 2016. Changing markets - Medicinal plants in the markets of La Paz and El Alto, Bolivia. Journal of Ethnopharmacology 193: 76-95.

Bye RA, Linares E. 1983. The role of plants found in the Mexican markets and their importance in ethnobotanical studies. Journal of Ethnobiology 3(1):1-13.

Centre for Disease Control and Prevention-CDC. 201). Global health - Ghana. https://www.cdc.gov/globalhealth/countries/ghana/default.htm (Accessed 19/10/2021).

Cory W. 2020. Package 'ethnobotany R' Version 0.1.7. Cran.R-Project.Org/Web/Packages/ ethnobotany R (Accessed 25/09/2021).

Cunningham AB. 2001. Applied ethnobotany: people, wild plant use and conservation. Earthscan Publishers, London, UK.

Dogor GK, Nyarko, RA, Anning AK, Oteng-Yeboah AA. 2018. Medicinal plant use and conservation practices by communities in the Togo Plateau Forest Reserve, Ghana. Journal of Medicinal Plants Research 12:575-589.

Friedman J, Yaniv Z, Dafni A, Palewitch D. 1986. A preliminary classification of the healing potential of medicinal plants, based on a rational analysis of an ethnopharmacological field survey among Bedouins in the Negev desert, Israel. Journal of Ethnopharmacology 16(2-3):275-287. 
Ghana Statistical Services 2014. 2010 Population and housing census. District analytical report: Ho Municipality.https://www2.statsghana.gov.gh/docfiles/2010_District_Report/Volta/HO\%20MUNICIPAL. pdf (Accessed 24/05/2021).

Heinrich M, Edwards S, Moerman DE, Leonti M. 2009. Ethnopharmacological field studies: A critical assessment of their conceptual basis and methods. Journal of Ethnopharmacology 124(1):1-17.

Idu M, Erhabor JO, Efijuemue HM. 2010. Documentation on medicinal plants sold in markets in Abeokuta, Nigeria. Tropical Journal of Pharmaceutical Research 9:110-118.

Irvine FR. 1961. Woody plants of Ghana. Oxford University Press, London, UK.

Konlan KD, Baku EA, Japiong M, Konlan KD, Doat AR, Suuk AN, Amoah RM. 2020. Practices of adults in a periurban community of the Ho Municipality on prevention of hypertension. International Journal of Hypertension. Article ID 2136213.

Martin GJ. 1995. Ethnobotany: A methods manual. Springer, Boston, USA.

Mary DA, Franco FM, Babu V. 2011. Assessing the contribution of local and traded biodiversity in community health care: a case study from Keelakodankulam village, South India. Ethnobotany Research and Applications, 9: 275-286.

Mshana N, Abbiw K, Addae-Mensah I, Adjanohoun E, Ahyi MRA, Ekpere KA, Enow-Orock EG, Gbile ZO, Noamesi GK, Odei MA, Odunlami H, Oteng-Yeboah AA, Sarpong K, Sofowora A, Tackie AN. 2000. Traditional medicine and pharmacopoeia. Contribution to the revision of ethnobotanical and floristic studies in Ghana. Accra, Ghana: Organization of African Unity/Scientific, Technical \&amp; Research Commission.

Obiri BD, Addai A, 2007. A survey of economic botanicals on the Kumasi Central Market Ghana. Journal of Forestry 21/22:50-71.

Okagu IU, Ndefo JC, Aham EC, Udenigwe CC 2021. Zanthoxylum Species: A comprehensive review of traditional uses, phytochemistry, pharmacological and nutraceutical applications. Molecules 26(13):4023.

Oliveira FCS, Vieira FJ, Amorim AN, Barros RFM 2021. The use and diversity of medicinal flora sold at the open market in the city of Oeiras, semiarid region of Piauí, Brazil. Ethnobotany Research and Applications 22:119.

Prance GT, Balee W, Boom BM, Carneiro RL. 1987. Quantitative ethnobotany and the case for conservation in Amazonia. Conservation Biology 1(4):296-310.

Ouedraogo L, Endl J, Sombié PAED, Schaefer H, Kiendrebeogo M. 2020. Ethnobotanical use and conservation assessment of medicinal plants sold in markets of Burkina Faso. Ethnobotany Research and Applications 20 1-25.

Quiroz D, Towns A, Legba SI, Swier J, Brière S, Sosef M, van Andel T. 2014. Quantifying the domestic market in herbal medicine in Benin, West Africa. Journal of Ethnopharmacology 151(3):1100-1108.

Tardío J, Pardo-de-Santayana M. 2007. Cultural importance indices: a comparative analysis based on the useful wild plants of southern Cantabria (Northern Spain). Economic Botany 62:24-39.

Thomas E, Vandebroek I, Sanca S, Van Damme P. 2009. Cultural significance of medicinal plant families and species among Quechua farmers in Apillapampa, Bolivia. Journal of Ethnopharmacology 122(1):60-67.

Tinitana F, Rios M, Romero-Benavides JC, de la Cruz Rot M, Pardo-de-Santayana M. 2016. Medicinal plants sold at traditional markets in southern Ecuador. Journal of Ethnobiology and Ethnomedicine 12(1):29.

Towns AM, Quiroz D, Guinee, L, de Boer H, van Andel T. 2014. Volume, value and floristic diversity of Gabon's medicinal plant markets. Journal of Ethnopharmacology 155(2):1184-1193.

University of Ghana. 2018. State of the nation's health report 2018. https://publichealth.ug.edu.gh/sites/publichealth.ug.edu.gh/files/docs/state_of_the_nations_interior_final_compre ssed-compressed_2.pdf (Accessed 19/10/2021).

van Andel T, Behari-Ramdas J, Havinga R, Groenendijk S. 2007. The medicinal plant trade in Suriname. Ethnobotany Research and Applications 5:351-372.

van Andel TR, Myren BJ, van Onselen S. 2012. Ghana's herbal market. Journal of Ethnopharmacology 140:368-378. Williams VL, Balkwill K, Witkowski ETF 2000. Unraveling the commercial market for medicinal plants and plant parts on the Witwatersrand, South Africa. Economic Botany 54(3)310-327. 